\title{
The progeny of stellar dynamics and stellar evolution within an $N$-body model of NGC 188
}

\author{
Aaron M. Geller, ${ }^{1}$ Jarrod R. Hurley ${ }^{2}$ and Robert D. Mathieu ${ }^{1}$ \\ ${ }^{1}$ University of Wisconsin-Madison, 475 N. Charter Street, Madison WI 53706, USA \\ ${ }^{2}$ Swinburne University of Technology, P.O. Box 218, Hawthorn, VIC 3122, Australia
}

\begin{abstract}
We present a direct $N$-body simulation modeling the evolution of the old (7 Gyr) open cluster NGC 188. This is the first $N$-body open cluster simulation whose initial binary population is directly defined by observations of a specific open cluster: M35 (150 Myr). We compare the simulated color-magnitude diagram at 7 Gyr to that of NGC 188, and discuss the blue stragglers produced in the simulation. We compare the solar-type main-sequence binary period and eccentricity distributions of the simulation to detailed observations of similar binaries in NGC 188. These results demonstrate the importance of detailed observations in guiding $N$-body open cluster simulations. Finally, we discuss the implications of a few discrepancies between the NGC 188 model and observations and suggest a few methods for bringing $N$-body open cluster simulations into better agreement with observations.
\end{abstract}

Keywords. open clusters and associations: individual (NGC 188), binaries: spectroscopic, blue stragglers, $N$-body simulations

\section{Introduction}

Recently, sophisticated $N$-body simulations have incorporated stellar dynamics and stellar evolution self-consistently, gaining the ability to accurately model open-clustersized populations $\left(N \sim 10^{4}\right)$ of single and binary stars through many billions of years. (e.g., NBODY6, Aarseth 2003; with stellar and binary evolution included by Hurley et al. (2000, 2002). Concurrently, large observational surveys of open clusters, like the WIYN Open Cluster Study (wocs; Mathieu 2000), are coming to maturity, providing comprehensive databases of open cluster characteristics, including detailed information on their binary populations. These observations, and specifically those of the binaries, provide important tests and guidance for $N$-body simulations. In this paper, we describe our first steps in using this wealth of data to help guide an $N$-body simulation with the aim of creating a realistic model of NGC 188 .

\section{2. $N$-body model of NGC 188}

The groundbreaking Hurley et al. (2005) model of M67 is a clear example of the potential for $N$-body simulations to model real open clusters, and thereby address longstanding questions about the evolution of a binary population, origins of blue stragglers (BSs), and specific observed characteristics of a given open cluster. Hurley et al. had very limited knowledge of the real binary population in M67, and thus aimed to match other important observables like the number of BSs, the spatial profile, etc. Today, through the wocs survey, work at the Harvard-Smithsonian Center for Astrophysics (e.g., Latham 2006) and others, we know a great deal about the M67 binaries. It is now clear that, although Hurley et al. (2005) were successful in matching many observed characteristics 

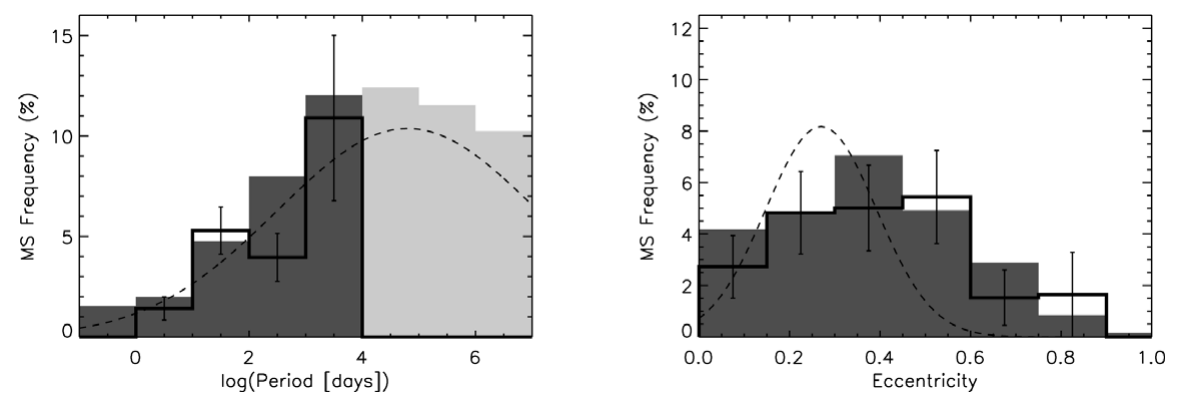

Figure 1. Binary period (left) and eccentricity (right) frequency distributions, comparing the primordial binary population in the simulation (filled histograms) to the observed main-sequence binary population of M35 (150 Myr; solid black lines). The M35 observed distributions have been corrected for incompleteness to show all binaries with periods, $P<10^{4}$ days (excluding those with periods below the circularization period in the eccentricity distribution plot). The primordial binary distributions used in the simulation are plotted with dark gray for observationally detectable binaries $\left(P<10^{4}\right.$ days $)$, and light gray for longer-period binaries. We also show the Duquennoy \& Mayor (1991) Galactic-field binary frequency distributions as the dashed lines for comparison.

of M67, the binary frequency at 4 Gyr was too high and the simulation contained too many short-period binaries throughout the evolution of the cluster (see Geller \& Mathieu, in prep.). The binary population has a significant effect on the formation mechanisms and rates of BSs as well as the dynamical evolution of the entire cluster. Therefore, correctly modeling the binary population is one of the most important ingredients in creating a realistic open cluster model.

The old (7 Gyr) open cluster NGC 188, like M67 (4 Gyr), has a rich and well-studied binary population (e.g., Geller et al. 2009a; Geller \& Mathieu, in prep.), a large population of BSs (Mathieu \& Geller 2009), and a wealth of observations from the wocs group and others (see Geller et al. 2008). Indeed, NGC 188 is quickly becoming one of the most best-studied and most important open clusters for understanding the origins of $\mathrm{BSs}$, the characteristics of an evolved binary population and the dynamical state of an evolved open cluster. These data provide ideal and much-needed guidance for an $N$-body simulation of NGC 188 .

\subsection{Simulation method}

All simulations were run using the NBODY6 code (Aarseth 2000), with slight modifications to define the initial binary population and output format. All stellar evolution is incorporated as in Hurley et al. (2000) and binary evolution, including tides, mass transfer, etc., is treated as in Hurley et al. (2002). BSs are modeled in the same manner as in Hurley et al. (2005). The simulations were executed on the supercomputer at the Swinburne Centre for Astrophysics and Supercomputing.

The NGC 188 simulation was initialized with a mass of $23870 \mathrm{M}_{\odot}(N=24000$ stars $)$ chosen from a Kroupa (2001) initial mass function and distributed according to a Plummer profile. We assume solar metallicity for the stars in the model (Sarajedini et al. 1999) and place the cluster in orbit around a point-mass Galactic potential according to the observed orbit of NGC 188 (Carraro \& Chiosi 1994). In the following, we discuss the binary population of the model in some detail, focusing on the period and eccentricity distributions and leaving more thorough discussions of methods and results for future papers. 

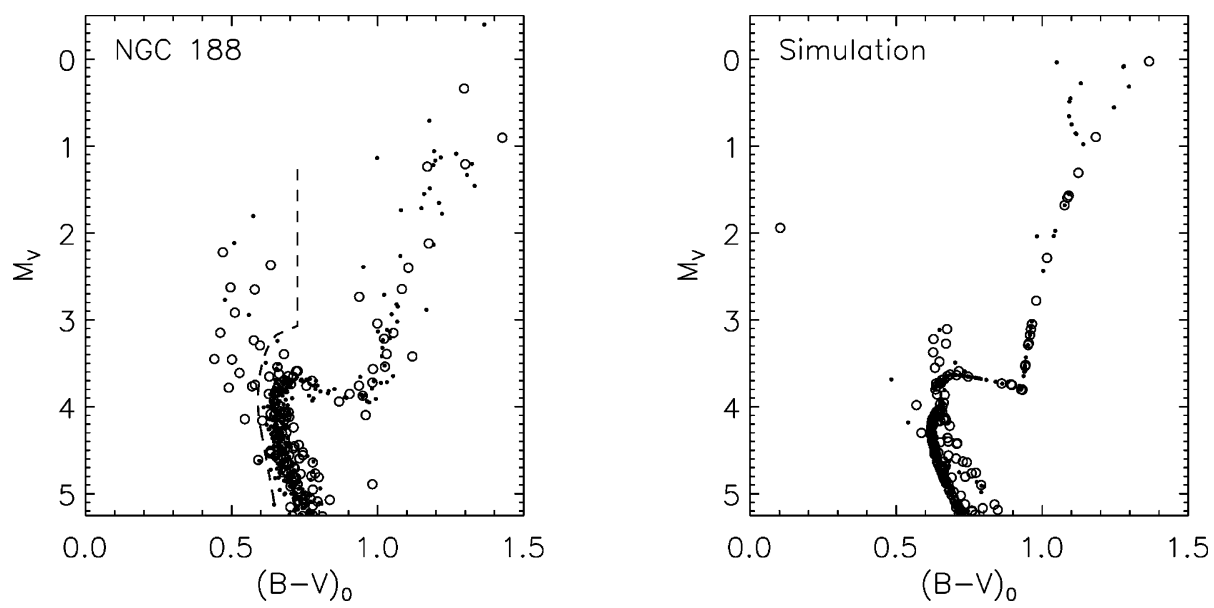

Figure 2. Color-magnitude diagrams of NGC 188 (left) and the NGC 188 simulation at 7 Gyr (right). The single stars are plotted as small black points, and the detected binaries are plotted using larger open circles. The NGC 188 BSs are identified as being to the left of the dashed line (as in Geller et al. 2008), and within the simulation BSs are identified as being main-sequence stars with masses greater than the turnoff mass (and reside as a similar region to observed BSs in NGC 188). The simulation succeeds as reproducing the overall form of the observed singleand binary-star sequence, but has not created the large number of observed BSs.

\subsection{Primordial binary population and observations of $M 35$}

The initial parameters defining a binary population have long been derived from theoretical analyses and predictions, since we lacked sufficiently detailed observations of young open cluster binary populations to help guide these choices. The wocs survey of M35 (Geller et al. 2009c) now allows for a major step forward. We can use these binaries as a proxy for the primordial binary population in this simulation. There is no a priori reason to presume that the M35 binaries will evolve into the NGC 188 binaries. Still, the detailed observations of the M35 binaries allow us to create the first $N$-body model whose initial binary population is directly defined by observations and to study the evolution of these binaries for the entire age of NGC 188 (7 Gyr).

M35 is observed to have a main-sequence (MS) binary frequency of $22 \pm 3 \%$ out to periods of $10^{4}$ days. We have chosen to initialize the simulation with a binary frequency of $25 \%$ out to the same period range. In Figure 1 we show the M35 incompletenesscorrected binary period and eccentricity distributions (black line), compared to the initial conditions used in the NGC 188 model (filled histograms). As we only detect binaries with periods $<10^{4}$ days, we use the Galactic-field binaries (Duquennoy \& Mayor 1991) to extend the period distribution to longer periods. Thus, we input a primordial binary population with a lognormal period distribution and a Gaussian eccentricity distribution.

\subsection{Comparing to observations of $N G C 188$}

The wocs observations of NGC 188 (Geller et al. 2008, 2009a; Geller \& Mathieu, in prep.) allow for a detailed comparison with the NGC 188 model. In the following analysis, we limit the output of our simulation to only include stars within the Geller et al. observable domain (in both mass and binary period). We first focus on the color-magnitude diagram (CMD) at 7 Gyr shown in Figure 2. Note that the stellar evolution incorporated into the simulation does an excellent job of reproducing the 'normal' single- and binary-star sequence compared to the observed CMD. However, the simulation is notably lacking in BSs: there are 21 observed BSs in NGC 188 and only 5 at 7 Gyr in the simulation. 

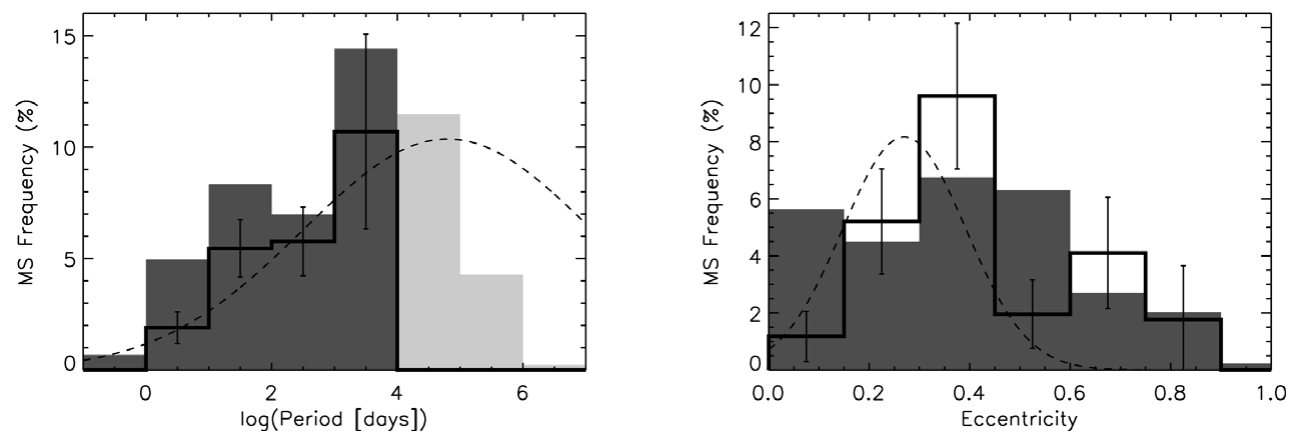

Figure 3. Period (left) and eccentricity (right) MS-binary frequency distributions at 7 Gyr. The NGC 188 observed distributions are plotted in the solid black lines and have been corrected for incompleteness in the same manner as in Figure 1, showing all binaries with $P<10^{4}$ days and excluding those with periods below the circularization period in the eccentricity distribution plot. The simulated binary distributions are plotted in the filled histograms with the same gray-scale coding as in Figure 1. Again, we also show the Duquennoy \& Mayor (1991) Galactic-field binary frequency distributions in the dashed lines for comparison. Note the close agreement between the simulated and observed binaries in both distributions and binary frequency.

This low number of BSs stays relatively constant throughout the simulation, and is likely due to the initial binary population. We will discuss the implications of this result in Section 3 .

We find the solar-type MS binary population in the simulation at 7 Gyr to correspond remarkably well to observations of similar binaries in NGC 188. In Figure 3 we compare the observed incompleteness-corrected NGC 188 binaries (black lines) to those of the simulation at 7 Gyr (filled histograms) in both period and eccentricity. The form of both the simulated distributions, as well as the binary frequency agree well with the observations of NGC 188 as well as the Galactic field (dashed lines; Duquennoy \& Mayor 1991). This close agreement is a testament to the power of using detailed observations to define the initial binary population in an $N$-body simulation.

Still, there are a few discrepancies between the simulated binary population and the observations. The circularization period in the model is significantly shorter than the observed value for NGC 188 (Figure 4). Meibom \& Mathieu (2005) find a circularization period of $14.5_{-2.2}^{+1.4}$ days, while a similar analysis of the model shows a period of 2.5 days. Interestingly, we also see too many circular MS binaries in the simulation at 7 Gyr (first bin in Figure 3), most having periods greater than the circularization period of both the simulation and observations. We discuss both of these results in the following section.

\section{Discussion and future directions}

By using the observed M35 binaries as a proxy for our primordial binary population, we have managed to nearly reproduce the NGC 188 binaries within our $N$-body simulation at 7 Gyr. However, as noted in Section 2.3, the current NGC 188 model is deficient in BSs. Interestingly, the Hurley et al. (2005) model of M67 was able to produce the large number of observed BSs (though not their binary frequency), but was unable to reproduce the observed binary population. The quantity of BSs produced in the M67 model was largely dependent on the initial binary population. Hurley et al. found that a large fraction of short-period binaries was necessary to create the observed numbers of BSs in M67 (and NGC 188). These short-period binaries not only have a higher likelihood of creating BSs in isolation (e.g., through mass transfer resulting from Roche-lobe overflow), 


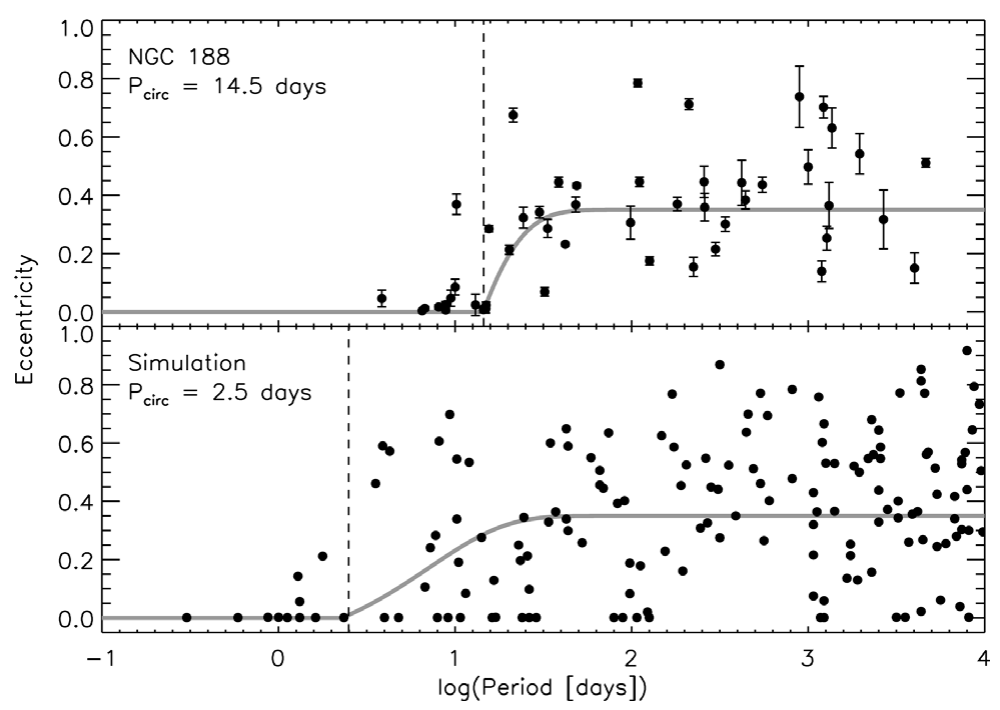

Figure 4. Eccentricity plotted against the logarithm of the period $(e-\log P)$ at 7 Gyr for the observed (top) and simulated (bottom) solar-type MS NGC 188 binaries. The solid gray lines represent the best-fit circularization functions from Meibom \& Mathieu (2005) for each sample, respectively. The tidal processes in the simulation result in a circularization period that is significantly lower than in NGC 188. Also, the simulation produces a population of circular binaries with $P \gg P_{\text {circ }}$ that is not observed in NGC 188 (nor in the Galactic field). Most of these circular binaries in the model have previously gone through a stage of mass transfer.

but dynamical interactions involving short-period binaries have a higher probability of resulting in collisions and mergers (Fregeau et al. 2004). Observations of M35 binaries are very different from the flat period distribution and large binary frequency used in the M67 model (e.g., Figure 1). The important next step will be to match both the binary population and the BS population within one cohesive simulation. We are currently exploring a number of possible methods for addressing this issue, the most promising of which appears to be the inclusion of primordial triples (e.g., Kozai-induced mergers; Perets \& Fabrycky 2009, larger cross section for dynamical interactions, etc.).

The tidal circularization period is one of our best observational tools to study the effects of tides on a binary population. In Figure 4 and Section 2.3 we show that the circularization period at $7 \mathrm{Gyr}$ in the simulation is significantly lower than what is observed in NGC 188. This suggests that the tidal-energy-dissipation rate may be underestimated in the $N$-body model (at least for solar-type MS binaries). We may also (or alternatively) require a form of pre-MS tidal circularization (e.g., Kroupa 1995). As pointed out by Mathieu (2008), tidal processes have a significant impact on dynamical interactions between single and binary stars, and the energy dissipation rates can be the deciding factor between a close fly-by, tidal capture or even a merger or collision that could result in a BS. Wocs data (e.g., Meibom \& Mathieu 2005) provide the ideal foundation from which we will improve the tidal physics within the $N$-body model.

Finally, the simulation has an excess of circular binaries with periods beyond the circularization period. The majority of these have undergone some form of mass transfer, which has quickly circularized the orbit. However, similar populations of circular binaries at $P \gg P_{\text {circ }}$ are not observed in open clusters nor in the Galactic field (e.g., Duquennoy \& Mayor 1991; Meibom \& Mathieu 2005). This may suggest that mass transfer, especially in initially eccentric binaries, does not necessarily lead to circular binaries (e.g., Sepinsky et al. 2007; Bonačić Marinović et al. 2008), or that these cases of mass transfer happen 
much more frequently in the simulation than in reality. Correctly modeling the products of mass transfer and common-envelope evolution is critical to accurately reproduce BSs as well as the binary population within an $N$-body simulation.

We will present further details about this NGC 188 model in future papers. Additionally, we will explore the various methods mentioned above for bringing the NGC 188 model, and $N$-body simulations in general, in better agreement with observations. This synergy between observations and simulations will continue to refine the $N$-body method and reveal further insights into the origins of BSs, the evolution of a binary population and the dynamical evolution of star clusters.

\section{Acknowledgements}

This work was funded in part by a National Science Foundation (NSF) East Asia and Pacific Summer Institutes (EAPSI) fellowship, the Wisconsin Space Grant Consortium and NSF grant AST-0406615.

\section{References}

Aarseth, S. J. 2000, in: V. G. Gurzadyan \& R. Ruffini (eds.), The Chaotic Universe, Proc. Second ICRA Network Workshop, Adv. Ser. Astrophys. Cosmol., vol. 10, p. 286

Aarseth, S. J. 2003, Gravitational N-Body Simulations, Cambridge: Cambridge University Press Bonačić Marinović, A. A., Glebbeek E., \& Pols, O. R. 2008, A\&̈A, 480, 797

Carraro, G. \& Chiosi, C. 1994, A\& $A, 288,751$

Duquennoy, A. \& Mayor, M. 1991, AA, 248, 485

Fregeau, J. M., Cheung, P., Portegies Zwart, S. F., \& Rasio, F. A. 2004, MNRAS, 352, 1

Geller, A. M., Mathieu, R. D., Braden, E. K., Meibom, S., Platais, I. \& Dolan, C. 2009b, AJ, submitted

Geller, A. M., Mathieu, R. D., Harris, H. C., \& McClure, R. D. 2008, AJ, 135, 2264

Geller, A. M., Mathieu, R. D., Harris, H. C., \& McClure, R. D. 2009a, AJ, 137, 3743

Hurley, J. R., Pols, O. R., Aarseth, S. J., \& Tout, C. A. 2005, MNRAS, 363, 293

Hurley, J. R., Pols, O. R., \& Tout, C. A. 2002, MNRAS, 329, 897

Hurley, J. R., Tout, C. A., \& Pols, O. R. 2000, MNRAS, 315, 543

Kroupa, P. 1995, MNRAS, 277, 1507

Kroupa, P. 2001, MNRAS, 322, 231

Latham, D. W. 2006, in: K. A. van der Hucht (ed.) Highlights Astron. vol. 14, p. 444

Mathieu, R. D. 2000, ASP Conf. Ser., 198, 517

Mathieu, R. D. 2008, in: E. Vesperini, M. Giersz \& A. Sills (eds.), Dynamical Evolution of Dense Stellar Systems, Proc. IAU Symp. No. 246, 79

Mathieu, R. D. \& Geller, A. M. 2009, Nature, in press

Meibom, S. \& Mathieu, R. D. 2005, ApJ, 620, 970

Mermilliod, J.-C., Rosvick, J. M., Duquennoy, A., \& Mayor, M. 1992, A $6 A$, 265, 513

Perets, H. B. \& Fabrycky, D. C. 2009, ApJ, 697, 1048

Sarajedini, A., von Hippel, T., Kozhurina-Platais, V., \& Demarque, P. 1999, AJ, 118, 2894

Sepinsky, J. F., Willems, B., Kalogera, V., \& Rasio, F. A. 2007, ApJ, 667, 1170 